\title{
Efecto de la aplicación de plasma seminal sobre la tasa de preñez en alpacas con diferentes tiempos de monta
}

\section{Effect of the application of seminal plasma on the pregnancy rate in alpacas with different copula times}

\author{
Renato Sánchez P. ${ }^{1}$, Wilfredo Huanca L., ${ }^{1,3}$, Jesús Turín V. ${ }^{1}$, Aida Cordero R. ${ }^{2}$
}

\section{Resumien}

\begin{abstract}
El objetivo de este estudio fue determinar el efecto del tiempo de la cópula y de la aplicación intramuscular de plasma seminal (PS) en alpacas sobre la tasa de preñez. Se utilizaron 161 alpacas hembras con tiempo de descanso posparto $\geq 15$ días, con cría al pie, y con presencia de folículos preovulatorios $\geq 7 \mathrm{~mm}$, identificados mediante ultrasonografía. Los animales se distribuyeron aleatoriamente en seis grupos: $\mathrm{G} 1(\mathrm{n}=28) 5$ min de cópula sin aplicación de PS; G2 (n=28) 5 min de cópula con aplicación de $1 \mathrm{ml}$ de PS i.m.; G3 $(\mathrm{n}=27) 10$ min de cópula sin aplicación de PS; G4 ( $\mathrm{n}=27) 10$ min de cópula más $1 \mathrm{ml}$ de PS; G5 $(n=26)>15$ min de cópula sin PS; G6 ( $n=25)>15$ min de cópula con $1 \mathrm{ml}$ PS. Para evitar el efecto macho se utilizó al mismo animal para la monta de una hembra del grupo del PS y otra del control por cada tiempo de estudio. El diagnóstico de preñez se hizo mediante ultrasonografía transrectal a los 25 días de la monta, obteniéndose 50.0, 64.3, 63.0, 70.4, 76.9 y $80.0 \%$ de preñez para G1, G2, G3, G4, G5 y G6 respectivamente. Los resultados señalan una mejor tasa de preñez $(p<0.05)$ de $G 6$ respecto a $G 1, G 2, G 3$ y G4, pero similar a G5. El tiempo de cópula $\geq 15$ minutos permitió una mejor tasa de preñez $(p<0.05)$ respecto a tiempos de 5 y 10 minutos. La aplicación de plasma seminal no mejoró significativamente la tasa de preñez a los 25 días de la cópula.
\end{abstract}

Palabras clave: alpacas; tasa de preñez; plasma seminal; tiempo de empadre; FIO

\section{Abstract}

The aim if this study was to determine the effect of copulation time and the intramuscular application of seminal plasma (PS) in alpacas on pregnancy rate. A total of 161 female alpacas with $\geq 15$ days postpartum, with calf at foot, and with the presence of

\footnotetext{
${ }^{1}$ Laboratorio de Reproducción Animal, Facultad de Medicina Veterinaria, Universidad Nacional Mayor de San Marcos, Lima, Perú

${ }^{2}$ Departamento de Nutrición, Facultad de Zootecnia, Universidad Nacional Agraria La Molina, Lima, Perú

${ }^{3}$ E-mail: whuancal@unmsm.edu.pe

Proyecto $N^{\circ}$ 405-PNICP-PIAP-2014
}

Recibido: 27 de abril de 2018

Aceptado para publicación: 2 de febrero de 2019 
preovulatory follicle $\geq 7 \mathrm{~mm}$, identified by ultrasonography were used. The animals were randomized into six groups: $\mathrm{G} 1(\mathrm{n}=28) 5$ min of copula without PS application; $\mathrm{G} 2(\mathrm{n}=28)$ $5 \mathrm{~min}$ of copula with application of $1 \mathrm{ml}$ of PS i.m.; $\mathrm{G} 3(\mathrm{n}=27) 10 \mathrm{~min}$ of copula without application of PS; G4 ( $n=27) 10$ min of copula plus $1 \mathrm{ml}$ of PS; G5 $(n=26)>15$ min of copula without PS; G6 $(n=25)>15$ min of copula with $1 \mathrm{ml}$ PS. To avoid the male effect, the same animal was used to mate a female from the PS group and another from the control group for each study time. Pregnancy diagnosis was done by transrectal ultrasonography 25 days after mating, obtaining 50.0, 64.3, 63.0, 70.4, 76.9 and $80.0 \%$ of pregnancy for $\mathrm{G} 1$, $\mathrm{G} 2, \mathrm{G} 3, \mathrm{G} 4, \mathrm{G} 5$ and G6 respectively. The results indicate a better pregnancy rate $(\mathrm{p}<0.05)$ of G6 with respect to G1, G2, G3 and G4, but similar to G5. The copulation time $\geq 15$ minutes allowed a better pregnancy rate $(\mathrm{p}<0.05)$ compared to times of 5 and 10 minutes. The application of seminal plasma did not significantly improve the pregnancy rate 25 days post-copula.

Key words: alpacas; pregnancy rate; seminal plasma; mating time; OIF

\section{INTRODUCCIÓN}

La situación actual de la crianza de los camélidos sudamericanos (CSA) sugiere la necesidad de un programa de mejora genética, orientado a seleccionar y multiplicar a los mejores animales desde un punto de vista productivo, ejemplares con un diámetro de fibra comprendido entre 18 a $21 \mu \mathrm{m}$, cuyo número aún es bastante reducido. Sin embargo, la baja eficiencia reproductiva de estos animales limita considerablemente las posibilidades de difundir este material genético en el conjunto de la población (Huanca, 2008).

Los índices de fertilización a los tres días pos-servicio son superiores al $85 \%$, pero los porcentajes de alpacas que mantienen la gestación a los 30 días del servicio son mucho menores, estimándose una pérdida embrionaria que puede llegar hasta el 50\% (Fernández-Baca et al., 1970).

El mayor conocimiento sobre los mecanismos de ovulación en los CSA se inició con el reporte sobre la capacidad del plasma seminal de machos vasectomizados para in- ducir la ovulación en alpacas (Ríos, 1989), En estudios posteriores se demuestra el potente efecto luteotrópico de este factor, mediante el incremento de las concentraciones circulantes de LH y el cambio rápido en la vascularización del folículo preovulatorio y el cuerpo lúteo en desarrollo (Ulloa-Leal et al., 2014; Ratto et al., 2005).

Este factor inductor de ovulación (FIO) fue demostrado al aplicar plasma seminal en forma intramuscular para obtener un $93 \mathrm{y}$ $90 \%$ de ovulaciones en alpacas y llamas, respectivamente (Adams et al., 2005) y 100\% de ovulaciones en llamas y alpacas (Ratto et al., 2006). Así mismo, se ha demostrado que la vía intramuscular después de la cópula es la mejor ruta para inducir ovulación (Ratto et al., 2005).

La inducción de ovulación con la aplicación de plasma seminal conteniendo el FIO y la necesidad de maximizar el uso de machos de alto valor genético motivó a desarrollar el presente estudio que tuvo como objetivo evaluar el efecto de la aplicación de plasma seminal sobre la tasa de preñez en alpacas, con diferentes tiempos de monta. 
Cuadro 1. Distribución de los grupos de estudio considerados para las actividades experimentales

\begin{tabular}{ccl}
\hline Grupo & $\begin{array}{c}\text { Alpacas } \\
(\mathrm{n})\end{array}$ & Tratamiento \\
\hline 1 & 28 & 5 minutos de cópula \\
2 & 28 & 5 minutos de cópula $+1 \mathrm{ml}$ de plasma seminal IM \\
3 & 27 & 10 minutos de cópula \\
4 & 27 & 10 minutos de cópula $+1 \mathrm{ml}$ de plasma seminal IM \\
5 & 26 & Tiempo de cópula normal $(>15 \mathrm{~min})$ \\
6 & 25 & Tiempo de cópula normal $(>15 \mathrm{~min})+1 \mathrm{ml}$ de plasma seminal IM \\
\hline Total & 161 & \\
\hline
\end{tabular}

\section{Materiales y Métodos}

\section{Lugar de Estudio}

El estudio se realizó entre enero y marzo de 2015 en el Centro de Investigación y Producción Quimsachata de la Estación Experimental ILLPA, Instituto Nacional de Innovación Agraria (INIA-Puno), ubicado a 4100 msnm, en el distrito de Cabanillas, Puno, Perú. La precipitación pluvial anual en la zona oscila entre 400 y $688 \mathrm{~mm}$, siendo los meses más húmedos los comprendidos entre diciembre y marzo.

\section{Unidades Experimentales}

Se utilizaron 161 hembras, con edades entre 5 y 7 años, procedentes de la punta de hembras en lactación con cría al pie. Los animales tenían un tiempo de descanso $\geq 15$ días. Además, se emplearon 80 machos de comprobada eficacia reproductiva. Las hembras eran alimentadas a base de pasturas naturales y estaban separadas en grupos (llamadas puntas), sin contacto con el plantel de machos reproductores, que solo se movilizaban a la zona de empadre en fechas programadas.

\section{Obtención del Plasma Seminal}

El plasma seminal (PS) fue obtenido mediante colecciones de semen realizadas a cinco alpacas machos adultos del Laboratorio de Reproducción Animal de la Facultad de Medicina Veterinaria de la Universidad Nacional Mayor de San Marcos, en Lima, Perú. Los machos tenían entre 6 a 8 años y presentaban características seminales óptimas. La colección de semen fue realizada con una vagina artificial de ovinos, pero adaptada para las alpacas, dos veces por semana y durante dos meses (aproximadamente 12 eyaculados por animal). Las muestras de semen colectadas por día de todos los machos se mezclaban y se procesaban de acuerdo con los procedimientos descritos por Adams et al. (2005).

Cada mezcla de eyaculados se diluyó $1: 1$ ( $\mathrm{vol} / \mathrm{vol})$ con buffer fosfato salino (PBS, Gibco, EEUU), y se hicieron pasajes en jeringas de $1.0 \mathrm{ml}$ para reducir la viscosidad. Luego se centrifugaron durante 30 minutos a $1500 \mathrm{~g}$ a temperatura ambiente, para eliminar la fracción celular del semen. El sobrenadante fue recuperado y una gota se evaluó por microscopía para confirmar la ausencia de espermatozoides o cualquier resto 
celular (se volvía a centrifugar si aparecían espermatozoides). El plasma seminal libre de espermatozoides se almacenó en tubos de 3 $\mathrm{ml}$, agregando gentamicina $10 \mu \mathrm{g} / 10 \mathrm{ml}$, y se llevó a congelación a $-20^{\circ} \mathrm{C}$ para su almacenamiento y traslado hasta su uso.

\section{Tratamientos}

Las hembras fueron seleccionadas por receptividad con un macho celador y separadas en un corral. Luego fueron evaluadas mediante ecografía transrectal para confirmar la presencia de un folículo preovulatorio $\geq 7 \mathrm{~mm}$ y distribuidas en corrales para ser cubiertas por los machos.

Las hembras fueron asignadas por sorteo en seis grupos, según el tiempo de cópula $(5,10,>15$ minutos $)$, con y sin aplicación intramuscular de plasma seminal (Cuadro 1). Además, se utilizaron 28 machos donde cada macho empadró a una hembra del grupo plasma seminal y a otra del grupo control por cada tiempo de cópula del estudio. Los servicios de cópula se realizaron en diferentes días, dividiéndose las hembras receptivas presentes entre los tratamientos en estudio. Los machos realizaban dos servicios por día en el caso de los grupos 1, 2, 3 y 4, pero un solo servicio en los grupos 5 y 6 , dejando levantarse al macho si cumplía al menos los 15 minutos, pero que no excediera de 18 minutos. El periodo total de servicios fue de 3 semanas.

\section{Diagnóstico de Gestación}

Se realizó a los 25 días de la monta mediante ecografía transrectal y en base al criterio de determinar la presencia de estructuras compatibles con un embrión y la presencia de los sacos gestacionales (Parraguez et al., 1996). El procedimiento se realizó con un ecógrafo Aloka SSD 500 (Japón) y un transductor lineal de $5.0 \mathrm{MHz}$, teniendo el mayor cuidado y evitando prolongar la manipulación de los animales para evitar estrés innecesario.

\section{Análisis Estadístico}

Se utilizó el programa $S A S \circledR$ System v. 9.4 (SAS Inc. Cary, USA) para el análisis de los datos. Los supuestos de distribución normal no se cumplieron en la variable respuesta (preñez), por lo que se transformó logarítmicamente el valor obtenido para cada tratamiento. Posteriormente, se analizó mediante un modelo lineal general con factores fijos: tiempo de cópula (5, 10 y 15 minutos) y plasma seminal (aplicación y no aplicación). Además, se consideró la interacción entre estas variables en el modelo. Se utilizó la media de mínimos cuadrados para identificar los tratamientos estadísticamente diferentes. Se consideró que las diferencias observadas eran estadísticamente significativas cuando $\mathrm{p}<0.05$.

\section{Resultados}

La tasa de preñez en los seis grupos experimentales se muestra en el Cuadro 2, en tanto que las tasas de preñez según los tiempos de cópula $(5,10,>15$ minutos) o la aplicación (sí, no) intramuscular de plasma seminal se muestran en los cuadros 3 y 4 , respectivamente. La mayor tasa de preñez se presentó en G6 (e»15 min de cópula con aplicación de PS) $(\mathrm{p}<0.05)$ respecto a los otros grupos experimentales, pero similar a G5 que tuvo similar tiempo de monta y sin aplicación de PS. No se observó diferencia significativa entre los otros grupos experimentales (Cuadro 2).

El Cuadro 3 presenta la tasa de preñez según tiempo de cópula, registrándose una mejor tasa de preñez cuando el tiempo de cópula fue $\geq 15$ minutos $(\mathrm{p}<0.05)$. Por otro lado, la tasa de preñez por efecto de la aplicación de PS no mostró diferencia significativa con los grupos donde no se aplicó la PS (Cuadro 4). 
Cuadro 2. Tasa de preñez (\%) en alpacas servidas con tres tiempos de monta y con o sin aplicación intramuscular de plasma seminal

\begin{tabular}{cccccc}
\hline Tratamiento & $\begin{array}{c}\text { Tiempo de } \\
\text { cópula }\end{array}$ & $\begin{array}{c}\text { Plasma } \\
\text { seminal }\end{array}$ & $\begin{array}{c}\text { Hembras } \\
\text { servidas (n) }\end{array}$ & $\begin{array}{c}\text { Hembras } \\
\text { preñadas }(\mathrm{n})\end{array}$ & $\begin{array}{c}\text { Tasa de preñez } \\
(\%)\end{array}$ \\
\hline $\mathrm{G}_{1}$ & 5 & No & 28 & 14 & $50.0^{\mathrm{a}}$ \\
$\mathrm{G}_{2}$ & 5 & Sí & 28 & 18 & $64.3^{\mathrm{a}}$ \\
$\mathrm{G}_{3}$ & 10 & No & 27 & 17 & $63.0^{\mathrm{a}}$ \\
$\mathrm{G}_{4}$ & 10 & Sí & 27 & 19 & $70.4^{\mathrm{ab}}$ \\
$\mathrm{G}_{5}$ & $>15$ & No & 26 & 20 & $76.9^{\mathrm{cb}}$ \\
$\mathrm{G}_{6}$ & $>15$ & Sí & 25 & 20 & $80.0^{\mathrm{c}}$ \\
\hline
\end{tabular}

abc Superíndices diferentes dentro de columna indican diferencia significativa $(p<0.05)$

Cuadro 3. Tasa de preñez (\%) en alpacas según tiempos de cópula

\begin{tabular}{cccc}
\hline $\begin{array}{c}\text { Tiempo de cópula } \\
\text { (minutos) }\end{array}$ & $\begin{array}{c}\text { Número de hembras } \\
\text { empadradas }\end{array}$ & $\begin{array}{c}\text { Número de hembras } \\
\text { preñadas }\end{array}$ & $\begin{array}{c}\text { Tasa de preñez } \\
(\%)\end{array}$ \\
\hline 5 & 56 & 32 & $57.14^{\mathrm{a}}$ \\
10 & 54 & 36 & $66.67^{\mathrm{a}}$ \\
$>15$ & 51 & 40 & $78.43^{\mathrm{b}}$ \\
\hline
\end{tabular}

${ }^{a b}$ Superíndices diferentes dentro de columna indican diferencia significativa $(p<0.05)$

Cuadro 4. Tasa de preñez (\%) en alpacas según la aplicación o no de plasma seminal

\begin{tabular}{cccc}
\hline Plasma seminal & $\begin{array}{c}\text { Número de hembras } \\
\text { empadradas }\end{array}$ & $\begin{array}{c}\text { Número de hembras } \\
\text { preñadas }\end{array}$ & $\begin{array}{c}\text { Tasa de preñez } \\
(\%)\end{array}$ \\
\hline Con aplicación & 80 & 57 & $71.25^{\mathrm{a}}$ \\
Sin aplicación & 81 & 51 & $62.96^{\mathrm{a}}$ \\
\hline
\end{tabular}

a Superíndices iguales dentro de columna indican que no existe diferencia significativa

\section{Discusión}

Diversos estudios han señalado que las alpacas, como especie de ovulación inducida, requieren del estímulo de la cópula para ovular; no obstante, se conoce que la ovulación en alpacas es inducida por la presencia en el semen de un componente proteico denominado Factor Inductor de Ovulación (FIO) (Sumar et al., 1993; Adams et al., 2005).

La administración de plasma seminal/ FIO intramuscular incrementa las concentraciones plasmáticas de LH (Ulloa-Leal et al., 2014) seguido de ovulación en los camélidos 
sudamericanos domésticos, reportándose hasta un $100 \%$ de ovulaciones en alpacas (Palián, 2010; López et al., 2012;) y llamas (Adams et al., 2005; Ulloa-Leal et al., 2014). El FIO presenta un potente efecto luteotrópico, incrementando la vascularización durante la fase preovulatoria y las primeras horas del cuerpo lúteo (Ulloa-Leal et al., 2014). Este factor inductor de la ovulación podría favorecer el empadre aumentando las concentraciones de $\mathrm{P}_{4}$ producida por el cuerpo lúteo, que ayudarían al desarrollo del embrión (Turín, 2015).

En el estudio se reportan diferencias estadísticas en tasa de preñez de animales que recibieron PS y que tuvieron más de 15 minutos de cópula (Cuadro 2) y, en general, entre los tiempos de cópula mayor a $15 \mathrm{mi}$ nutos respecto a 5 y 10 minutos de cópula. Esta diferencia puede ser explicada por la presencia del FIO presente en el semen y el mayor tiempo de cópula, acción que contribuye a una mayor irritación de la mucosa uterina, favoreciendo posiblemente a una mayor absorción del FIO, según lo sugiere Panez et al. (2009); asimismo, el efecto luteotrópico del FIO contribuye a una mayor producción de progesterona y a una mejor tasa de sobrevivencia embrionaria, según lo sugiere Turín (2015).

En el presente trabajo no se observó diferencia significativa en la tasa de preñez a los 25 días de la monta por efecto de la aplicación del plasma seminal (Cuadro 4). No obstante, Turín (2015) demostró que este factor ejerce una actividad favorable para el mantenimiento de la preñez como agente luteotrópico, favoreciendo el desarrollo del cuerpo lúteo y el mantenimiento de la preñez en comparación a la aplicación de GnRH o monta natural sola.

\section{Conclusiones}

- El tiempo de cópula en la alpaca influye de manera significativa sobre la tasa de preñez obteniendo mejores resultados en las cópulas de 15 minutos a más.
- La aplicación de plasma seminal intramuscular no influyó sobre la tasa de preñez a los 25 días.

\section{Literatura Citada}

1. Adams G, Ratto M, Huanca W, Singh J. 2005. Ovulation-inducing factor in the seminal plasma of alpacas and llamas. Biol Reprod 73: 452-457. doi: 10.1095/ biolreprod.105.040097

2. Fernández Baca S, Hansel W, Novoa C. 1970. Embryonic mortality in the alpaca. Biol Reprod 3: 243-251. doi: 10.1093/biolreprod/3.2.243

3. Huanca T. 2008. Efecto de la administración de gonadotropinas exógenas (FSH y eCG) en la respuesta ovárica y la producción de embriones en alpacas (Vicugna pacos). Tesis Doctoral. España: Univ. de Santiago de Compostela. $142 \mathrm{p}$.

4. Palián J. 2010. Inducción de ovulación con plasma seminal o análogo de $\mathrm{GnRH}$ (acetato de buserelina) y su efecto sobre la tasa de concepción en alpacas (Vicugna pacos), inseminadas con semen fresco. Tesis de Médico Veterinario. Lima: Univ. Nacional Mayor de San Marcos. 66 p.

5. Panez S, Huanca W, Huanca T, Ratto M, Adams G 2009. Efecto del sitio de deposición del plasma seminal sobre la tasa de ovulación y formación del cuerpo lúteo en alpacas. Rev Inv Vet Perú 20: 21-27. doi: 10.15381/rivep.v20i1.526

6. Parraguez V, Gazitua F, Raggi L. 1996. Estudio ultrasonográfico de la gestación en alpacas (Lama pacos): Resultados preliminares. Rev Argentina Prod Anim Bs As 16: 337-340.

7. Ratto M, Huanca W, Singh J, Adams, G 2005. Local versus systemic effect of ovulation-inducing factor in the seminal plasma of alpacas. Reprod Biol Endocrinol 3 (29). doi:10.1186/14777827-3-29 
8. Ratto MH, Huanca W, Singh J, Adams GP. 2006. Comparison of the effect of ovulation-inducing factor (OIF) in the seminal plasma of llamas, alpacas, and bulls. Theriogenology 66: 1102-1106. doi: 10.1016/j.theriogenology.2006.02.050

9. Ríos M. 1989. Presencia de un factor de inducción de la ovulación en el semen de alpaca y toro. Tesis de Médico Veterinario. Lima: Univ. Nacional Mayor de San Marcos. 30 p.

10. Sumar J, Bravo PW, Foote WC. 1993. Sexual receptivity and time of ovulation- in alpacas. Small Ruminant Res 11: 143150. doi: 10.1016/0921-4488(93)90147-A

11. Turín J. 2015. Efecto de la aplicación intramuscular de plasma seminal sobrela supervivencia embrionaria en alpacas. Tesis de Médico Veterinario. Lima: Univ. Nacional Mayor de San Marcos. 49 p.

12. Ulloa-Leal C, Bogle O, Adams G Ratto M. 2014. Luteotrophic effect of ovulationinducing factor/nerve growth factor present in the seminal plasma of 1lamas. Theriogenology 81: 1101-107. doi: 10.1016/ j.theriogenology.2014.-01.038 